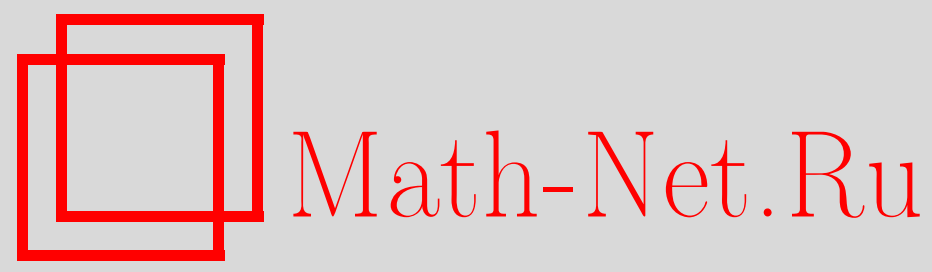

А. Н. Уриновский, Оценка норм операторов умножения в гильбертовых алгебрах, Матем. заметки, 2002, том 72, выпуск 2, 283-291

DOI: https://doi.org/10.4213/mzm422

Использование Общероссийского математического портала Math-Net.Ru подразумевает, что вы прочитали и согласны с пользовательским соглашением http://www.mathnet.ru/rus/agreement

Параметры загрузки:

IP : 54.164 .48 .24

26 апреля 2023 г., $07: 04: 21$

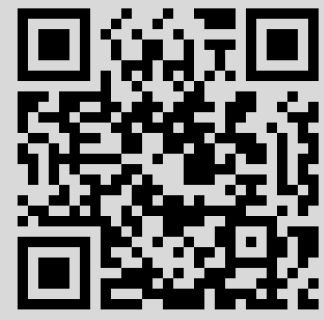




\section{ОЦЕНКА НОРМ ОПЕРАТОРОВ УМНОЖЕНИЯ В ГИЛЬБЕРТОВЫХ АЛГЕБРАХ}

\section{А. Н. Уриновский}

В работе для билинейного оператора, задаваемого операцией умножения в произвольной ассоциативной алгебре $\mathbf{V}$ с единицей $\mathbf{e}_{0}$ над полем $\mathbb{R}$ или $\mathbb{C}$, доказано, что точная нижняя грань его норм относительно всех скалярных произведений в этой алгебре, нормирующих е 0 , либо бесконечна, либо не превосходит $\sqrt{4 / 3}$. Найдены достаточные условия для того, чтобы эта грань была не меньше $\sqrt{4 / 3}$. Конечность этой грани для бесконечномерных грассмановых алгебр впервые была доказана Купшем и Смоляновьм (использована для построения функционального представления фоковских супералгебр).

Библиография: 1 название.

1. Основные определения. Пусть задана $\mathbf{V}, \circ, \mathbf{e}_{0}-$ ассоциативная алгебра с единицей над полем $\mathbb{K}(\mathbb{K}=\mathbb{R}, \mathbb{C})$ с операциями $(+, \circ)$.

Алгебру $\mathbf{V}$ можно рассматривать одновременно как векторное пространство, поэтому элементы алгебры мы будем называть также векторами. На алгебре V, рассматриваемой как векторное пространство, может быть введено скалярное произведение, обозначаемое символом $\langle\cdot, \cdot\rangle$, возможно с индексом в виде греческой буквы, например $\langle\cdot, \cdot\rangle_{\tau}$, тот же индекс будет указьваться и для нормы вектора при этом скалярном произведении. Однако если это не влечет затруднений, индекс мы можем опускать. Выделим множество скалярных произведений $\mathfrak{T}=\left\{\langle\cdot, \cdot\rangle_{\tau}:\left\|\mathbf{e}_{0}\right\|_{\tau}=1\right\}$. Далее мы рассматриваем только скалярные произведения из этого множества.

Для каждого скалярного произведения $\langle\cdot, \cdot\rangle_{\tau}$ определим функцию $P_{\tau}: \mathbf{V} \times \mathbf{V} \rightarrow \mathbb{R}_{0}^{+}$, где $\mathbb{R}_{0}^{+}$- множество неотрицательных действительных чисел, следующим образом:

$$
P_{\tau}\left(\mathbf{v}_{1}, \mathbf{v}_{2}\right)= \begin{cases}\frac{\left\|\mathbf{v}_{1} \circ \mathbf{v}_{2}\right\|_{\tau}}{\left\|\mathbf{v}_{1}\right\|_{\tau} \cdot\left\|\mathbf{v}_{2}\right\|_{\tau}} & \text { при }\left\|\mathbf{v}_{1}\right\|_{\tau} \cdot\left\|\mathbf{v}_{2}\right\|_{\tau} \neq 0 \\ 0 & \text { при }\left\|\mathbf{v}_{1}\right\|_{\tau} \cdot\left\|\mathbf{v}_{2}\right\|_{\tau}=0 .\end{cases}
$$

ЗАмечание 1. Для всех $\alpha, \beta \in \mathbb{K} \backslash\{0\}$ выполнено $P_{\tau}\left(\alpha \cdot \mathbf{v}_{1}, \beta \cdot \mathbf{v}_{2}\right)=P_{\tau}\left(\mathbf{v}_{1}, \mathbf{v}_{2}\right)$.

Введем еще обозначения:

$$
P_{\tau 0}=\sup _{\mathbf{v}_{1}, \mathbf{v}_{2} \in \mathbf{V}}\left(P_{\tau}\left(\mathbf{v}_{1}, \mathbf{v}_{2}\right)\right), \quad P_{0}=\inf _{\tau \in \mathfrak{T}}\left(P_{\tau 0}\right) .
$$

Число $P_{\tau 0}$ назовем мультипликативной нормой алгебры $\mathbf{V}$ при скалярном произведении $\tau$, а $P_{0}$ - мультипликативной нормой алгебры $\mathbf{V}$. Легко видеть, что $P_{\tau 0}-$ 
норма билинейного оператора, задаваемого операцией “о”, при скалярным произведении $\langle\cdot, \cdot\rangle_{\tau}$.

В данной работе мы оцениваем возможные значения $P_{0}$. Из определения следует, что $P_{0}$ не зависит от конкретного скалярного произведения и может считаться характеристикой самой алгебры.

Если не оговорено противное, то будем считать, что на алгебре $\mathbf{V}$ уже задано некоторое скалярное произведение $\langle\cdot, \cdot\rangle_{\tau} \in \mathfrak{T}$.

2. Вспомогательные утверждения. Выделим подмножество элементов из V c индексом нильпотентности 2:

$$
\mathbf{N}=\{\mathbf{f} \in \mathbf{V}: \mathbf{f} \circ \mathbf{f}=\mathbf{0}\}
$$

ПРЕДЛОЖЕНИЕ 1. Если $\mathbf{N} \neq \varnothing$, mo $P_{\tau 0} \geqslant \sqrt{4 / 3}$. Ecли же существует $\mathbf{f} \in \mathbf{N}$ такой, что $\left\langle\mathbf{e}_{0}, \mathbf{f}\right\rangle \neq 0$, то неравенство строгое.

ДокАЗАТЕЛЬСтво. Можно считать, что $\|\mathbf{f}\|=1$. Выберем $\alpha \in \mathbb{K}$ такое, что

$$
|\alpha|=\frac{1}{\sqrt{2}} \quad \text { и } \quad\left\langle\mathbf{e}_{0}, \alpha \mathbf{f}\right\rangle \in \mathbb{R}, \quad\left\langle\mathbf{e}_{0}, \alpha \mathbf{f}\right\rangle \leqslant 0 .
$$

Рассмотрим

$$
P_{\tau}\left(\mathbf{e}_{0}+\alpha \mathbf{f}, \mathbf{e}_{0}+\alpha \mathbf{f}\right)=\frac{\left\|\mathbf{e}_{0}+2 \alpha \mathbf{f}\right\|}{\left\|\mathbf{e}_{0}+\alpha \mathbf{f}\right\|^{2}}=\frac{\sqrt{\left\langle\mathbf{e}_{0}, \mathbf{e}_{0}\right\rangle+4\left\langle\mathbf{e}_{0}, \alpha \mathbf{f}\right\rangle+4\langle\alpha \mathbf{f}, \alpha \mathbf{f}\rangle}}{\left\langle\mathbf{e}_{0}, \mathbf{e}_{0}\right\rangle+2\left\langle\mathbf{e}_{0}, \alpha \mathbf{f}\right\rangle+\langle\alpha \mathbf{f}, \alpha \mathbf{f}\rangle}
$$

Возводя это выражение в квадрат и подставляя известные значения, получаем

$$
P_{\tau}^{2}\left(\mathbf{e}_{0}+\alpha \mathbf{f}, \mathbf{e}_{0}+\alpha \mathbf{f}\right)=\frac{1+4\left\langle\mathbf{e}_{0}, \alpha \mathbf{f}\right\rangle+2}{\left(1+2\left\langle\mathbf{e}_{0}, \alpha \mathbf{f}\right\rangle+1 / 2\right)^{2}}=\frac{4}{3+4\left\langle\mathbf{e}_{0}, \alpha \mathbf{f}\right\rangle} \geqslant \frac{4}{3}
$$

(равенство достигается только при $\left.\left\langle\mathbf{e}_{0}, \alpha \mathbf{f}\right\rangle=0\right)$.

Условия предложения могут быть несколько обобщены, однако ниже нам это не понадобится. Предложение является усилением аналогичного результата из [1].

ПРЕДЛОЖЕНИЕ 2. Если существуют $\mathbf{f}_{1}, \mathbf{f}_{2} \in \mathbf{N}$ такие, что $\mathbf{f}_{1} \circ \mathbf{f}_{2}=\mathbf{f}_{2} \circ \mathbf{f}_{1} \neq \mathbf{0}$, mo $P_{\tau 0}>\sqrt{4 / 3}$.

ДокАЗАТЕЛЬСТво. Если хотя бы один из указанных векторов не ортогонален $\mathbf{e}_{0}$, то доказательство предложения вытекает из предложения 1 , поэтому рассмотрим только случай, когда $\mathbf{f}_{1}, \mathbf{f}_{2} \perp \mathbf{e}_{0}$. Очевидно, что для всех $\alpha, \beta \in \mathbb{R}$ выполнено $\left(\alpha \mathbf{f}_{1}+\beta \mathbf{f}_{2}\right) \perp \mathbf{e}_{0}$. Введем новые векторы

$$
\mathbf{v}=\mathbf{e}_{0}+\alpha \mathbf{f}_{1}+\beta \mathbf{f}_{2}, \quad \mathbf{u}=\alpha \mathbf{f}_{1}+\beta \mathbf{f}_{2}, \quad \mathbf{u}^{\prime}=\mathbf{f}_{1} \circ \mathbf{f}_{2} .
$$

Откуда

$$
\begin{gathered}
\mathbf{v} \circ \mathbf{v}=\mathbf{e}_{0}+2 \mathbf{u}+2 \alpha \beta \mathbf{u}^{\prime} \\
\|\mathbf{v} \circ \mathbf{v}\|^{2}=\left\|\mathbf{e}_{0}+2 \mathbf{u}\right\|^{2}+4 \alpha \beta \cdot \operatorname{Re}\left\langle\mathbf{e}_{0}, \mathbf{u}^{\prime}\right\rangle+8 \alpha \beta \cdot \operatorname{Re}\left\langle\mathbf{u}, \mathbf{u}^{\prime}\right\rangle+4 \alpha^{2} \beta^{2}\left\langle\mathbf{u}^{\prime}, \mathbf{u}^{\prime}\right\rangle
\end{gathered}
$$


Алгоритм нахождения коэффициентов следующий.

1. Примем $\alpha=1,|\beta|=1$.

2. Выберем знак для $\beta$ так, чтобы $4 \alpha \beta \cdot \operatorname{Re}\left\langle\mathbf{e}_{0}, \mathbf{u}^{\prime}\right\rangle \geqslant 0$.

3. Выберем знак для $\alpha$ (если он отрицательньй, то одновременно меняется и знак при $\beta$ ) так, чтобы $8 \alpha \beta \cdot \operatorname{Re}\left\langle\mathbf{u}, \mathbf{u}^{\prime}\right\rangle \geqslant 0$. Это всегда возможно, потому что $\operatorname{Re}\left\langle-\mathbf{u}, \mathbf{u}^{\prime}\right\rangle=$ $-\operatorname{Re}\left\langle\mathbf{u}, \mathbf{u}^{\prime}\right\rangle$.

4. Подбираем $\gamma>0$ так, чтобы

$$
\left\|\alpha_{0} \mathbf{f}_{1}+\beta_{0} \mathbf{f}_{2}\right\|=\frac{1}{\sqrt{2}}, \quad \text { где } \alpha_{0}=\frac{\alpha}{\gamma}, \quad \beta_{0}=\frac{\beta}{\gamma} .
$$

Отсюда следует, что

$$
\begin{gathered}
\|\mathbf{v} \circ \mathbf{v}\|^{2}=\left\|\mathbf{e}_{0}+2 \mathbf{u}\right\|^{2}+s, \quad s>0, \\
P_{\tau}\left(\mathbf{e}_{0}+\alpha_{0} \mathbf{f}_{1}+\beta_{0} \mathbf{f}_{2}, \mathbf{e}_{0}+\alpha_{0} \mathbf{f}_{1}+\beta_{0} \mathbf{f}_{2}\right)=\frac{\sqrt{3+s}}{3 / 2}>\sqrt{\frac{4}{3}} .
\end{gathered}
$$

ПРЕДЛОЖЕНИЕ 3. Если существуют $\mathbf{f}_{1}, \mathbf{f}_{2} \in \mathbf{N}$ такие, ито $\mathbf{f}_{1} \circ \mathbf{f}_{2}=-\mathbf{f}_{2} \circ \mathbf{f}_{1} \neq \mathbf{0}$, mo $P_{\tau 0}>\sqrt{4 / 3}$.

ДокАЗАТЕЛЬСтво. Пусть вектор $\mathbf{f}_{3}=\mathbf{f}_{1} \circ \mathbf{f}_{2}$. Так как оператор "о" ассоциативен, то $\mathbf{f}_{3} \in \mathbf{N}$. Действительно,

$$
\mathbf{f}_{3} \circ \mathbf{f}_{3}=\left(\mathbf{f}_{1} \circ \mathbf{f}_{2}\right) \circ\left(\mathbf{f}_{1} \circ \mathbf{f}_{2}\right)=-\left(\mathbf{f}_{1} \circ \mathbf{f}_{2} \circ \mathbf{f}_{2} \circ \mathbf{f}_{1}\right)=\mathbf{0} .
$$

Аналогично доказывается, что $\mathbf{f}_{1} \circ \mathbf{f}_{3}=\mathbf{f}_{3} \circ \mathbf{f}_{1}=\mathbf{f}_{2} \circ \mathbf{f}_{3}=\mathbf{f}_{3} \circ \mathbf{f}_{2}=\mathbf{0}$. Если хотя бы один из векторов $\mathbf{f}_{1}, \mathbf{f}_{2}, \mathbf{f}_{3}$ не ортогонален $\mathbf{e}_{0}$, то доказательство предложения вытекает из предложения 1 , поэтому рассмотрим только случай, когда $\mathbf{f}_{1}, \mathbf{f}_{2}, \mathbf{f}_{3} \perp \mathbf{e}_{0}$. Очевидно, что для всех $\alpha, \beta, \gamma \in \mathbb{K}$ вьполнено $\left(a \mathbf{f}_{1}+\beta \mathbf{f}_{2}+\gamma \mathbf{f}_{3}\right) \perp \mathbf{e}_{0}$.

Можно считать, что $\left\|\mathbf{f}_{1}\right\|=\left\|\mathbf{f}_{2}\right\|=1$. Построим вектор $\mathbf{u}_{0}=\beta \mathbf{f}_{2}+\gamma \mathbf{f}_{3}, \beta, \gamma \in \mathbb{R}$, удовлетворяющий следующим условиям:

1) $\beta \neq 0$;

2) $\operatorname{Re}\left\langle\mathbf{u}_{0}, \mathbf{f}_{3}\right\rangle \neq 0$

3) $\left\|\mathbf{u}_{0}\right\|=\sqrt{1 / 2}$.

Это всегда возможно. Рассмотрим

$$
P_{\tau}\left(\mathbf{e}_{0}+\mathbf{u}_{0}, \mathbf{e}_{0}+\mathbf{u}_{0}\right)=\sqrt{\frac{4}{3}} .
$$

Введем векторную функцию от действительного переменного $x$ :

$$
\mathbf{u}(x)=x \mathbf{f}_{1}+\beta \mathbf{f}_{2}+\gamma \mathbf{f}_{3},
$$

очевидно, $\mathbf{u}(0)=\mathbf{u}_{0}, \mathbf{u}(x)+\mathbf{u}(-x)=2 \mathbf{u}(0)$. Имеем

$$
\begin{aligned}
P_{\tau}\left(\mathbf{e}_{0}+\mathbf{u}(x), \mathbf{e}_{0}+\mathbf{u}(-x)\right) & =\frac{\left\|\mathbf{e}_{0}+\mathbf{u}(x)+\mathbf{u}(-x)+\mathbf{u}(x) \circ \mathbf{u}(-x)\right\|}{\left\|\mathbf{e}_{0}+\mathbf{u}(x)\right\| \cdot\left\|\mathbf{e}_{0}+\mathbf{u}(-x)\right\|} \\
& =\frac{\left\|\mathbf{e}_{0}+2 \mathbf{u}_{0}+2 \beta x \mathbf{f}_{3}\right\|}{\left\|\mathbf{e}_{0}+\mathbf{u}_{0}+x \mathbf{f}_{1}\right\| \cdot\left\|\mathbf{e}_{0}+\mathbf{u}_{0}-x \mathbf{f}_{1}\right\|} .
\end{aligned}
$$


Введем функцию

$$
F(x)=P_{\tau}^{2}\left(\mathbf{e}_{0}+\mathbf{u}(x), \mathbf{e}_{0}+\mathbf{u}(-x)\right),
$$

очевидно, $F(0)=4 / 3$. Функция представляет собой некую дробь. Раскроем числитель и знаменатель дроби и подставим известные значения:

$$
\begin{aligned}
\left\|\mathbf{e}_{0}+2 \mathbf{u}_{0}+2 \beta x \mathbf{f}_{3}\right\|^{2}= & \left\|\mathbf{e}_{0}+2 \mathbf{u}_{0}\right\|^{2}+4 \beta x \cdot \operatorname{Re}\left\langle\mathbf{e}_{0}, \mathbf{f}_{3}\right\rangle \\
& +8 \beta x \cdot \operatorname{Re}\left\langle\mathbf{u}_{0}, \mathbf{f}_{3}\right\rangle+4 \beta^{2} x^{2}\left\langle\mathbf{f}_{3}, \mathbf{f}_{3}\right\rangle \\
= & 3+8 \beta x \cdot \operatorname{Re}\left\langle\mathbf{u}_{0}, \mathbf{f}_{3}\right\rangle+4 \beta^{2} x^{2}\left\|\mathbf{f}_{3}\right\|^{2}
\end{aligned}
$$

Далее,

$$
\begin{aligned}
\left\|\mathbf{e}_{0}+\mathbf{u}_{0} \pm x \mathbf{f}_{1}\right\|^{2} & =\left\|\mathbf{e}_{0}+\mathbf{u}_{0}\right\|^{2} \pm 2 x \cdot \operatorname{Re}\left\langle\mathbf{e}_{0}, \mathbf{f}_{1}\right\rangle \pm 2 x \cdot \operatorname{Re}\left\langle\mathbf{u}_{0}, \mathbf{f}_{1}\right\rangle+x^{2}\left\langle\mathbf{f}_{1}, \mathbf{f}_{1}\right\rangle \\
& =\frac{3}{2}+x^{2} \pm 2 x \cdot \operatorname{Re}\left\langle\mathbf{u}_{0}, \mathbf{f}_{1}\right\rangle .
\end{aligned}
$$

Отсюда

$$
\left\|\mathbf{e}_{0}+\mathbf{u}_{0}+x \mathbf{f}_{1}\right\|^{2} \cdot\left\|\mathbf{e}_{0}+\mathbf{u}_{0}-x \mathbf{f}_{1}\right\|^{2}=\left(\frac{3}{2}+x^{2}\right)^{2}-4 x^{2}\left(\operatorname{Re}\left\langle\mathbf{u}_{0}, \mathbf{f}_{1}\right\rangle\right)^{2}
$$

Отсюда получаем следующее выражение для $F(x)$ :

$$
F(x)=\frac{3+8 \beta x \cdot \operatorname{Re}\left\langle\mathbf{u}_{0}, \mathbf{f}_{3}\right\rangle+4 \beta^{2} x^{2}\left\|\mathbf{f}_{3}\right\|^{2}}{\left(3 / 2+x^{2}\right)^{2}-4 x^{2}\left(\operatorname{Re}\left\langle\mathbf{u}_{0}, \mathbf{f}_{1}\right\rangle\right)^{2}}
$$

Найдем производную функции в точке $x=0$ (она существует):

$$
F^{\prime}(0)=\frac{8 \beta \cdot \operatorname{Re}\left\langle\mathbf{u}_{0}, \mathbf{f}_{3}\right\rangle \cdot 9 / 4}{(9 / 4)^{2}}=\frac{32}{9} \beta \cdot \operatorname{Re}\left\langle\mathbf{u}_{0}, \mathbf{f}_{3}\right\rangle \neq 0
$$

Следовательно, при $x=0$ функция $F(x)$ не достигает максимума, а значит, при некотором $x_{0}$ выполнено $F\left(x_{0}\right)>F(0)$. Из этого следует, что при $\alpha=x_{0}$

$$
P_{\tau}\left(\mathbf{e}_{0}+\alpha \mathbf{f}_{1}+\beta \mathbf{f}_{2}+\gamma \mathbf{f}_{3}, \mathbf{e}_{0}-\alpha \mathbf{f}_{1}+\beta \mathbf{f}_{2}+\gamma \mathbf{f}_{3}\right)>\sqrt{\frac{4}{3}}
$$

Резюмируя полученные результаты, мы можем дать следующую оценку: если $\mathbf{N} \neq \varnothing$, то при любом скалярном произведении $\langle\cdot, \cdot\rangle_{\tau}$ выполнено $P_{\tau 0} \geqslant \sqrt{4 / 3}$, если к тому же существуют два элемента алгебры $\mathbf{f}_{1}, \mathbf{f}_{2} \in \mathbf{N}$ такие, что $\mathbf{f}_{1} \circ \mathbf{f}_{2}= \pm \mathbf{f}_{2} \circ \mathbf{f}_{1} \neq \mathbf{0}$, то неравенство будет строгим. 
Лемма 1. Пусть

$$
\mathbf{u}=\sum_{m=1}^{\infty} \mathbf{u}_{m} \quad u \quad \mathbf{v}=\sum_{n=1}^{\infty} \mathbf{v}_{n}, \quad \mathbf{u}_{m}, \mathbf{v}_{n} \neq \mathbf{0},
$$

где все векторы $\mathbf{u}_{m}$ ортогональны между собой, так же как и векторы $\mathbf{v}_{n}$ между собой. Пусть также

$$
\mathbf{u}_{m} \circ \mathbf{v}_{n}=\sum_{j=1}^{\infty} \mathbf{w}_{m, n}^{j}, \quad \mathbf{u} \circ \mathbf{v}=\sum_{m, n, j=1}^{\infty} \mathbf{w}_{m, n}^{j},
$$

при этом сумма последнего ряда не зависит от порядка әлементов. Далее, все пары $(m, n)$ разбиты на непустые непересекающиеся классы $\mathfrak{A}_{1}^{1}, \mathfrak{A}_{2}^{1}, \ldots$ таким образом, что для любого $\mathfrak{A}_{k}^{1}$ существует вектор

$$
\mathbf{g}_{k}^{1}=\sum_{(m, n) \in \mathfrak{A}_{k}^{1}} \mathbf{w}_{m, n}^{1}, \quad \mathbf{g}_{k 1}^{1} \perp \mathbf{g}_{k 2}^{1} \quad n p u \quad k_{1} \neq k_{2} .
$$

Точно так же пары разбиты для любого верхнего индекса $j$ наклассы $\mathfrak{A}_{1}^{j}, \mathfrak{A}_{2}^{j}, \ldots$, при этом разбиение на классы по каждому верхнему индексу не зависит от разбиений по другим верхним индексам.

Тогда имеет место оценка

$$
P_{\tau}(\mathbf{u}, \mathbf{v}) \leqslant \sum_{j=1}^{\infty} \sup _{k} \sqrt{\sum_{(m, n) \in \mathfrak{A}_{k}^{j}} \frac{\left\|\mathbf{w}_{m, n}^{j}\right\|^{2}}{\left\|\mathbf{u}_{m}\right\|^{2} \cdot\left\|\mathbf{v}_{n}\right\|^{2}}}
$$

ДокАЗАТЕЛЬСтво. Сразу же отметим, что

$$
\|\mathbf{u}\| \cdot\|\mathbf{v}\|=\sqrt{\sum_{m, n=1}^{\infty}\left\|\mathbf{u}_{m}\right\|^{2} \cdot\left\|\mathbf{v}_{n}\right\|^{2}}, \quad\left\|\sum_{k} \mathbf{g}_{k}^{j}\right\|=\sqrt{\sum_{k}\left\|\mathbf{g}_{k}^{j}\right\|^{2}} .
$$

Пусть

$$
t_{j}=\sup _{k} \frac{\left\|\mathbf{g}_{k}^{j}\right\|}{\sqrt{\sum_{(m, n) \in \mathfrak{A}_{k}^{j}}\left\|\mathbf{u}_{m}\right\|^{2} \cdot\left\|\mathbf{v}_{n}\right\|^{2}}}
$$

тогда

$$
\sum_{k}\left\|\mathbf{g}_{k}^{j}\right\|^{2} \leqslant\left(t_{j}\right)^{2} \cdot \sum_{k} \sum_{(m, n) \in \mathfrak{A}_{k}^{j}}\left(\left\|\mathbf{u}_{m}\right\|^{2} \cdot\left\|\mathbf{v}_{n}\right\|^{2}\right)=\left(t_{j} \cdot\|\mathbf{u}\| \cdot\|\mathbf{v}\|\right)^{2},
$$

таким образом, $\left\|\sum_{k} \mathbf{g}_{k}^{j}\right\| /(\|\mathbf{u}\| \cdot\|\mathbf{v}\|) \leqslant t_{j}$. Далее,

$$
\begin{gathered}
\mathbf{u} \circ \mathbf{v}=\sum_{m, n=1}^{\infty} \mathbf{u}_{m} \circ \mathbf{v}_{n}=\sum_{m, n=1}^{\infty} \sum_{j=1}^{\infty} \mathbf{w}_{m, n}^{j}=\sum_{j=1}^{\infty} \sum_{m, n=1}^{\infty} \mathbf{w}_{m, n}^{j}=\sum_{j=1}^{\infty} \sum_{k} \mathbf{g}_{k}^{j}, \\
P_{\tau}(\mathbf{u}, \mathbf{v})=\frac{\left\|\sum_{j=1}^{\infty} \sum_{k} \mathbf{g}_{k}^{j}\right\|}{\|\mathbf{u}\| \cdot\|\mathbf{v}\|} \leqslant \sum_{j=1}^{\infty} \frac{\left\|\sum_{k} \mathbf{g}_{k}^{j}\right\|}{\|\mathbf{u}\| \cdot\|\mathbf{v}\|} \leqslant \sum_{j=1}^{\infty} t_{j} .
\end{gathered}
$$


Теперь осталось доказать, что

$$
\frac{\left\|\mathbf{g}_{k}^{j}\right\|^{2}}{\sum_{(m, n) \in \mathfrak{A}_{k}^{j}}\left\|\mathbf{u}_{m}\right\|^{2} \cdot\left\|\mathbf{v}_{n}\right\|^{2}} \leqslant \sum_{(m, n) \in \mathfrak{A}_{k}^{j}} \frac{\left\|\mathbf{w}_{m, n}^{j}\right\|^{2}}{\left\|\mathbf{u}_{m}\right\|^{2} \cdot\left\|\mathbf{v}_{n}\right\|^{2}}
$$

Введем сквозную нумерацию для пар, входящих в класс $\mathfrak{A}_{k}^{j}:$ пара $\left(m_{1}, n_{1}\right)$ встречается раньшепары $\left(m_{2}, n_{2}\right)$, если $m_{1}+n_{1}<m_{2}+n_{2}$ или $m_{1}+n_{1}=m_{2}+n_{2}$, но $m_{1}<m_{2}$. Тогда $\mathbf{g}_{k}^{j}=\sum_{i} \mathbf{w}_{i}$, где $\mathbf{w}_{i}=\mathbf{w}_{m, n}^{j}\left((m, n) \in \mathfrak{A}_{k}^{j}\right)$, соответствующее произведение $\left\|\mathbf{u}_{m}\right\| \cdot\left\|\mathbf{v}_{n}\right\|$ обозначим через $h_{i}$. Введем также обозначение $r_{i}=\left\|\mathbf{w}_{i}\right\| / h_{i}$, а значит, $\left\|\mathbf{w}_{i}\right\|=r_{i} \cdot h_{i}$. По неравенству Коши-Буняковского

$$
\left(\sum_{i} r_{i} \cdot h_{i}\right)^{2} \leqslant\left(\sum_{i} r_{i}^{2}\right) \cdot\left(\sum_{i} h_{i}^{2}\right)
$$

откуда

$$
\frac{\left(\sum_{i}\left\|\mathbf{w}_{i}\right\|\right)^{2}}{\sum_{i}\left(h_{i}\right)^{2}} \leqslant \sum_{i} \frac{\left\|\mathbf{w}_{i}\right\|^{2}}{\left(h_{i}\right)^{2}}
$$

а отсюда уже и вытекает (3).

Очевидно, что лемма верна и при $\mathbf{u}, \mathbf{v}$, состоящих из конечного числа векторов $\mathbf{u}_{m}$, $\mathbf{v}_{n}$. Если в лемме принять (а это всегда возможно) для всех $m, n \mathbf{u}_{m} \circ \mathbf{v}_{n}=\mathbf{w}_{m, n}^{1}$ и все пары $(m, n)$ принадлежат одному классу, то получается неравенство

$$
P_{\tau}(\mathbf{u}, \mathbf{v}) \leqslant \sqrt{\sum_{m, n=1}^{\infty} P_{\tau}^{2}\left(\mathbf{u}_{m}, \mathbf{v}_{n}\right)} \leqslant \sum_{m, n=1}^{\infty} P_{\tau}\left(\mathbf{u}_{m}, \mathbf{v}_{n}\right) .
$$

Если в условии леммы принять для всех $m, n \mathbf{u}_{m} \circ \mathbf{v}_{n}=\mathbf{w}_{m, n}^{1}$, то получается неравенство

$$
P_{\tau}(\mathbf{u}, \mathbf{v}) \leqslant \sup _{k} \sqrt{\sum_{(m, n) \in \mathfrak{A}_{k}^{1}} P_{\tau}^{2}\left(\mathbf{u}_{m}, \mathbf{v}_{n}\right)} \leqslant \sup _{k} \sum_{(m, n) \in \mathfrak{A}_{k}^{1}} P_{\tau}\left(\mathbf{u}_{m}, \mathbf{v}_{n}\right) .
$$

Если же, дополнительно, $\mathbf{w}_{m_{1}, n_{1}}^{1} \perp \mathbf{w}_{m_{2}, n_{2}^{1}}$ при $m_{1} \neq m_{2}$ или $n_{1} \neq n_{2}$, то

$$
P_{\tau}(\mathbf{u}, \mathbf{v}) \leqslant \sup _{1 \leqslant m, n<\infty} P_{\tau}\left(\mathbf{u}_{m}, \mathbf{v}_{n}\right)
$$

Заметим, что для этих формул уже необязательно $\mathbf{u}_{m}, \mathbf{v}_{n} \neq \mathbf{0}$.

3. Основная теорема. Основньп результатом этого пункта будет доказательство теоремы, показьвающей, что если значение $P_{0}$ конечно, то оно находится в промежутке $[1, \sqrt{4 / 3}]$.

ЛЕмма 2. Если $\mathbf{u}_{1}, \mathbf{u}_{2} \perp \mathbf{e}_{0}$, то для всех $\alpha, \beta \in \mathbb{K}$ выполнена оценка

$$
\left\|\alpha \beta \mathbf{e}_{0}+\alpha \mathbf{u}_{2}+\beta \mathbf{u}_{1}\right\| \leqslant \sqrt{\frac{4}{3}} \cdot\left\|\alpha \mathbf{e}_{0}+\mathbf{u}_{1}\right\| \cdot\left\|\beta \mathbf{e}_{0}+\mathbf{u}_{2}\right\| .
$$


ДокАЗАТЕльСтво. Допустим, мы подобрали такие $\alpha, \beta$, что

$$
\left\|\alpha \beta \mathbf{e}_{0}+\alpha \mathbf{u}_{2}+\beta \mathbf{u}_{1}\right\|>\sqrt{\frac{4}{3}} \cdot\left\|\alpha \mathbf{e}_{0}+\mathbf{u}_{1}\right\| \cdot\left\|\beta \mathbf{e}_{0}+\mathbf{u}_{2}\right\| .
$$

Обе части неравенства положительные, поэтому, возведя их в квадрат и используя неравенство треугольника, получаем

$$
\begin{gathered}
\left\|\alpha \beta \mathbf{e}_{0}+\alpha \mathbf{u}_{2}+\beta \mathbf{u}_{1}\right\|^{2}=|\alpha \beta|^{2}\left\|\mathbf{e}_{0}\right\|^{2}+\left\|\alpha \mathbf{u}_{2}+\beta \mathbf{u}_{1}\right\|^{2} \leqslant|\alpha \beta|^{2}+\left(\left\|\alpha \mathbf{u}_{2}\right\|+\left\|\beta \mathbf{u}_{1}\right\|\right)^{2}, \\
\left\|a \mathbf{e}_{0}+\mathbf{u}_{1}\right\|^{2}=|\alpha|^{2}+\left\|\mathbf{u}_{1}\right\|^{2}, \quad\left\|\beta \mathbf{e}_{0}+\mathbf{u}_{2}\right\|^{2}=|\beta|^{2}+\left\|\mathbf{u}_{2}\right\|^{2} .
\end{gathered}
$$

Следовательно, из (7) получается оценка

$$
|\alpha \beta|^{2}+\left(\left\|\alpha \mathbf{u}_{2}\right\|+\left\|\beta \mathbf{u}_{1}\right\|\right)^{2}>\frac{4}{3}\left(|\alpha|^{2}+\left\|\mathbf{u}_{1}\right\|^{2}\right) \cdot\left(|\beta|^{2}+\left\|\mathbf{u}_{2}\right\|^{2}\right) .
$$

Если одно из чисел $\alpha, \beta,\left\|\mathbf{u}_{1}\right\|,\left\|\mathbf{u}_{2}\right\|$ равно 0 , то очевидно, что допущенное неравенство не вьполняется, поэтому считаем их отличными от нуля.

Из (7) вытекает, что если $\left\|\mathbf{u}_{1}\right\|$ и $\alpha$ или же $\left\|\mathbf{u}_{2}\right\|$ и $\beta$ изменяются пропорционально, то неравенство сохраняется, поэтому будем считать, что $\left\|\mathbf{u}_{1}\right\|,\left\|\mathbf{u}_{2}\right\|=1$, тогда $\alpha$ и $\beta$ изменятся соответственно, но для простоты оставим для коэффициентов те же обозначения.

Полученная вьше формула преобразуется в следующую (так как правая часть неравенства больше нуля):

$$
\frac{(|\alpha| \cdot|\beta|)^{2}+(|\alpha|+|\beta|)^{2}}{\left(|\alpha|^{2}+1\right)\left(|\beta|^{2}+1\right)}>\frac{4}{3}
$$

откуда

$$
\frac{2|\alpha| \cdot|\beta|-1}{\left(|\alpha|^{2}+1\right)\left(|\beta|^{2}+1\right)}>\frac{1}{3}
$$

Введем новые переменные

$$
x=|\alpha| \cdot|\beta|, \quad y=\frac{|\alpha|}{|\beta|} \quad\left(\text { или }|\alpha|^{2}=x y, \quad|\beta|^{2}=\frac{x}{y}\right) .
$$

Очевидно, $y>0,2 x \geqslant 1$.

Введем вместо левой части неравенства (8) функцию от двух переменных $F(x, y)$ и оценим ее сверху:

$$
F(x, y)=\frac{2 x-1}{x^{2}+(y+1 / y) x+1} .
$$

Числитель дроби неотрищательный и не зависит от $y$, а знаменатель положительный и минимален при $y=1$. Отсюда вытекает

$$
F(x, y) \leqslant F(x, 1)=\frac{2 x-1}{(x+1)^{2}} .
$$

Несложно показать, что функция $F(x, 1)$ имеет максимум в точке $x=2$ и принимает при этом значение $F(2,1)=1 / 3$. Отсюда следует, что неравенство (8), а следовательно, и (7) невозможно. Таким образом, наше допущение неверно, а это значит, что лемма верна. 
СлЕДСТВИЕ 1. Если для любых двух векторов $\mathbf{u}_{1}, \mathbf{u}_{2}$, ортогональных $\mathbf{e}_{0}$, справедливо неравенство $P_{\tau}\left(\mathbf{u}_{1}, \mathbf{u}_{2}\right) \leqslant \varepsilon$, то $P_{\tau 0} \leqslant \sqrt{4 / 3}+\varepsilon ;$ в частности, если $\varepsilon=0$, mo $P_{\tau 0} \leqslant \sqrt{4 / 3}$.

Любой вектор $\mathbf{v} \in \mathbf{V}$ может быть представлен в виде

$$
\mathbf{v}=\alpha \mathbf{e}_{0}+\mathbf{u}, \quad \text { где } \alpha=\left\langle\mathbf{v}, \mathbf{e}_{0}\right\rangle, \quad \mathbf{u} \perp \mathbf{e}_{0} .
$$

Возьмем два любых ненулевых вектора $\mathbf{v}_{1}, \mathbf{v}_{2}$ :

$$
\mathbf{v}_{1}=\alpha_{1} \mathbf{e}_{0}+\mathbf{u}_{1}, \quad \mathbf{v}_{2}=\alpha_{2} \mathbf{e}_{0}+\mathbf{u}_{2}, \quad \alpha_{1}, \alpha_{2} \in \mathbb{K}, \quad \mathbf{u}_{1}, \mathbf{u}_{2} \perp \mathbf{e}_{0} .
$$

Имеем

$$
\begin{aligned}
P_{\tau}\left(\mathbf{v}_{1}, \mathbf{v}_{2}\right) & =\frac{\left\|\alpha_{1} \alpha_{2} \mathbf{e}_{0}+\alpha_{1} \mathbf{u}_{2}+\alpha_{2} \mathbf{u}_{1}+\mathbf{u}_{1} \circ \mathbf{u}_{2}\right\|}{\left\|\alpha_{1} \mathbf{e}_{0}+\mathbf{u}_{1}\right\| \cdot\left\|\alpha_{2} \mathbf{e}_{0}+\mathbf{u}_{2}\right\|} \\
& \leqslant \frac{\left\|\alpha_{1} \alpha_{2} \mathbf{e}_{0}+\alpha_{1} \mathbf{u}_{2}+\alpha_{2} \mathbf{u}_{1}\right\|}{\left\|\alpha_{1} \mathbf{e}_{0}+\mathbf{u}_{1}\right\| \cdot\left\|\alpha_{2} \mathbf{e}_{0}+\mathbf{u}_{2}\right\|}+P_{\tau}\left(\mathbf{u}_{1}, \mathbf{u}_{2}\right) \leqslant \sqrt{\frac{4}{3}}+\varepsilon
\end{aligned}
$$

ТЕОрема 1. Если на алгебре $\mathbf{V}$ задано скалярное произведение $\langle\cdot, \cdot\rangle_{\tau}$ с мультипликативной нормой $P_{\tau 0}=C$, где $C>\sqrt{4 / 3}$, то для любого полохительного $\varepsilon<C-\sqrt{4 / 3}$ существует скалярное произведение $\langle\cdot, \cdot\rangle_{\rho}$ с мультипликативной нормой $P_{\rho 0} \leqslant \sqrt{4 / 3}+\varepsilon$, причем для всех $\mathbf{v} \in \mathbf{V}$ выполнена оцен$\kappa a\|\mathbf{v}\|_{\rho} \leqslant(C / \varepsilon) \cdot\|\mathbf{v}\|_{\tau}$.

ДокАЗАТЕЛЬСтво. Согласно (9) мы можем представить $\mathbf{V}$ в виде прямой суммы двух ортогональных подпространств:

$$
\mathbf{V}=\mathbf{U}_{0} \oplus \mathbf{U}, \quad \text { где } \mathbf{U}_{0}=\left\{\alpha \mathbf{e}_{0}: \alpha \in \mathbb{K}\right\}, \quad \mathbf{U}=\left\{\mathbf{u}: \mathbf{u} \perp \mathbf{e}_{0}\right\} .
$$

Введем новое скалярное произведение $\langle\cdot, \cdot\rangle_{\rho}$, изменив нормы векторов из $\mathbf{U}:\|\mathbf{u}\|_{\rho}=$ $(C / \varepsilon) \cdot\|\mathbf{u}\|_{\tau}$, но сохранив свойства $\left\|\mathbf{e}_{0}\right\|_{\rho}=1$ и $\left\langle\mathbf{e}_{0}, \mathbf{u}\right\rangle_{\rho}=0$ для всех $\mathbf{u} \in \mathbf{U}$. Заметим, что норма любого вектора при замене скалярного произведения вырастет не более чем в $C / \varepsilon$ раз. Таким образом, для любых $\mathbf{u}_{1}, \mathbf{u}_{2} \perp \mathbf{e}_{0}$ получаем

$$
P_{\rho}\left(\mathbf{u}_{1}, \mathbf{u}_{2}\right)=\frac{\left\|\mathbf{u}_{1} \circ \mathbf{u}_{2}\right\|_{\rho}}{\left\|\mathbf{u}_{1}\right\|_{\rho} \cdot\left\|\mathbf{u}_{2}\right\|_{\rho}} \leqslant \frac{(C / \varepsilon) \cdot\left\|\mathbf{u}_{1} \circ \mathbf{u}_{2}\right\|_{\tau}}{(C / \varepsilon)^{2} \cdot\left\|\mathbf{u}_{1}\right\|_{\tau} \cdot\left\|\mathbf{u}_{2}\right\|_{\tau}} \leqslant \varepsilon
$$

откуда на основании следствия 1 и вытекает справедливость теоремы.

СлЕДСТВИЕ 2. Для ассочиативной алгебры с единичей справедлива альтернатива: либо $1 \leqslant P_{0} \leqslant \sqrt{4 / 3}$, либо $P_{0}=\infty$ (т.е. не существует скалярного произведения с конечной мультипликативной нормой).

Приведем пример алгебры со счетным базисом $\mathbf{e}_{0}, \mathbf{e}_{1}, \mathbf{e}_{2}, \ldots$, не имеющей конечной мультипликативной нормы. Зададим операцию “о” отдельно для четных и нечетных векторов: для любых $i, j$

$\mathbf{e}_{2 i} \circ \mathbf{e}_{2 j}=\mathbf{e}_{2 i+2 j}, \quad \mathbf{e}_{2 i+1} \circ \mathbf{e}_{2 j+1}=\mathbf{0}, \quad \mathbf{e}_{2 i+1} \circ \mathbf{e}_{2 j}=\mathbf{e}_{2 j} \circ \mathbf{e}_{2 i+1}=(2 i+1)^{2 j} \cdot \mathbf{e}_{2 i+1} ;$

тогда при любом $\langle\cdot, \cdot\rangle_{\tau}$ получаем

$$
P_{\tau}\left(\mathbf{e}_{2}, \mathbf{e}_{2 i+1}\right)=\frac{(2 i+1)^{2}}{\left\|\mathbf{e}_{2}\right\|_{\tau}} \underset{i \rightarrow \infty}{\longrightarrow} \infty
$$


ПримЕЧАнИЕ. Если бы отсутствовало требование нормированности единичного элемента, то "скачок" бы осуществлялся от 0 до $\infty$, однако приведенный пример был бы справедлив и в этом случае. Заметим также, что оператор умножения будет неограничен при любой введенной норме.

Приведем примеры использования теоремы 1.

Тривиальной мы назовем алгебру, состоящую только из элементов, пропорциональных единичному.

ПримеР 1. Мультипликативная норма произвольной конечномерной алгебры.

Пусть $\mathbf{V}$ - конечномерная алгебра, на которой задано скалярноепроизведение $\langle\cdot, \cdot\rangle_{\tau}$. Выберем произвольный ортонормированный базис $\mathbf{e}_{1}, \mathbf{e}_{2}, \ldots, \mathbf{e}_{n}$. Тогда для любых двух векторов $\mathbf{u}, \mathbf{v}$ вьполняется неравенство

$$
P_{\tau}(\mathbf{u}, \mathbf{v}) \leqslant \sum_{i, j=1}^{n} P_{\tau}\left(\mathbf{e}_{i}, \mathbf{e}_{j}\right)
$$

(см. (4) и замечание 1 ), откуда на основании следствия 2 получаем $1 \leqslant P_{0} \leqslant \sqrt{4 / 3}$. Можно показать, что мультипликативная норма $P_{0}$ может принимать лишь граничное значение указанного диапазона, причем $P_{0}=1$ только для тривиальной алгебры, а в случае алгебр над полем $\mathbb{R}$ - также для алгебр комплексных чисел и кватернионов.

ПримеР 2. Мультипликативная норма произвольной грассмановой алгебры.

Как было показано в [1], для произвольной грассмановой алгебры вьполнено $P_{0} \leqslant \sqrt{3}$ (в наших терминах). Отсюда из теоремы 1 следует, что $P_{0} \leqslant \sqrt{4 / 3}$, и из предложения 1 вытекает, что $P_{0}=\sqrt{4 / 3}$. Причем равенство $P_{\tau 0}=\sqrt{4 / 3}$ может быть достигнуто при каком-либо скалярном произведении $\langle\cdot, \cdot\rangle_{\tau}$ только при условии, что базис порождающего пространства состоит из одного вектора. Это следует из предложения 3 . Возможность равенства вытекает из следствия 1.

Автор выражает благодарность Н. Н. Шамарову за постановку задачи и постоянное внимание к работе.

\section{СПИСОК ЦИТИРОВАННОЙ ЛИТЕРАТУРЫ}

[1] Kupsh J., Smolyanov O. G. Hilbert norms for graded algebras // Proc. Amer. Math. Soc. 2000. V. 128. №6. P. 1647-1653. 